УДК 536.24:66.045.132

\title{
Аналитическое исследование теплопереноса в плотном слое гранулированного материала с внутренними источниками теплоты
}

\author{
И. Л. Боикова, А. В. Солодкая, Е. С. Бодюл, Э. И. Альтман \\ Одесская национальная академия пищевых технологий, ул. Канатная, 112, Одесса, 65039, Украина \\ E-mail: ${ }^{1}$ boshkova.irina@gmail.com, ${ }^{2}$ solodkaya140619@gmail.com, ${ }^{3}$ bodyulolena@ukr.net, ${ }^{4}$ ellaa@ukr.net \\ ORCID: ${ }^{1}$ http://orcid.org/0000-0001-5989-9223; ${ }^{2}$ http://orcid.org/0000-0002-4043-7667; \\ ${ }^{3}$ https://orcid.org/0000-0001-9925-434X; ${ }^{4}$ http://orcid.org/0000-0002-8934-2036
}

\begin{abstract}
Представлень результаты аналитического исследования тепломассопереноса в плотном слое гранулированного материала с газовым потоком как теплообменного участка регенеративных и рекуперативных устройств. Предложена математическая модель теплообмена между плотным слоем частиц и газовым потоком, полученная на основе двухкомпонентной гомогенной модели с учетом действия внутренних источников теплоты в одномерном приближении. Аналитическое исследование температурных полей газового и твердого компонентов при различных режимных и геометрических характеристиках показало удовлетворительное согласование расчетных и экспериментальных данных, однако следует учитывать чувствительность модели к заданным значениям коэффициентов теплоотдачи, неточность которых может привести к существенным ошибкам в расчете температурных полей.
\end{abstract}

Ключевые слова: Математическая модель; Теплообмін; Гранулированный слой; Одномерное приближение; Температурные зависимости; Внутренние источники; Расчет температурных полей.

\section{Аналітичне дослідження тепломасопереносу в щільному шарі гранульованого матеріалу з внутрішніми джерелами теплоти}

\author{
І. Л. Боикова, А. В. Солодка, О. С. Бодюл, Е. І. Альтман \\ Одеська національна академія харчових технологій, вул. Канатна, 112, Одеса, 65039, Україна
}

\begin{abstract}
Представлено результати аналітичного дослідження тепломасопереносу в щільному шарі гранульованого матеріалу з газовим потоком як теплообмінної ділянки регенеративних і рекуперативних пристроїв. Запропановано математичну модель теплообміну між щзільним шаром часток і газовим потоком, щзо отримана на основі двокомпонентної гомогенної моделі з урахуванням дії внутрішніх джерел теплоти в одномірному наближенні. Аналітичне дослідження температурних полів газового і твердого компонентів при різних режимних $i$ геометричних характеристиках показало задовільне узгодження розрахункових $і$ експериментальних даних, однак слід враховувати чутливість моделі до заданих значень коефіцієнтів тепловіддачі, неточність яких може привести до суттєвих помилок у розрахунку температурних полів.
\end{abstract}

Ключові слова: Математична модель; Теплообмін; Гранульований шар; Одномірне наближення; Температурні залежності; Внутрішні джерела; Розрахунок температурних полів.

(C) The Author(s) 2018. This article is an open access publication

This work is licensed under the Creative Commons Attribution 4.0 International License (CC BY) http://creativecommons.org/licenses/by/4.0/

\section{1. Введение}

Процессы нагрева, охлаждения, сушки, прокалки и термолиза дисперсных материалов широко используются в различных отраслях промышленности [1,2,3]. В отечественных и зарубежных исследованиях показана целесообразность ведения этих процессов в рекуперативных теплообменниках с плотным движущимся продуваемым и неподвижным слоем. Для разработки, расчета, проектирования и оптимизации таких теплообменников необходимо комплексное теоретическое и экспериментальное изучение тепломассопереноса в плотных слоевых системах $[4,5]$. Результаты таких ис- следований находят применение при тепловых расчетах различных устройств: установок энерготехнологической переработки топлива; химических ректоров; регенераторов катализатора; радиационных труб высокотемпературных печей для производства высокочистых окислов; сушилок для дисперсных материалов. Моделированию процессов теплообмена между газом и твердыми частицами уделяется значительное внимание вследствие важности знаний о влиянии отдельных факторов и условий на распределение температур и эффективность теплообмена[6.], [7]. Определение рациональных режимных параметров работы теплотехнических устройств также зависит от адекватной математической 
модели процесса теплообмена между потоками газа и гранулированного материала и надежных данных для коэффициентов межкомпонентных теплообмена, для получения которых требуются дополнительные экспериментальные исследования. Аналитические зависимости как результат решения математической модели нужны для отображения распределения температур в гранулированном материале и газе для предприятий с разным уровнем температуры и расходами газового потока, что позволит оптимизировать конструкторские решения при проектировании аппаратов в соответствии с целевыми направлениями. Задачей работы являлось проведение аналитических исследований теплопереноса в плотном слое гранулированного материала с внутренними источниками теплоты, которыми, в зависимости от принципиальных особенностей устройства, может быть теплота фазовых переходов, теплота преобразования энергии электромагнитного поля в тепловую, химические реакции.

Строгое описание процессов переноса в плотном неподвижном фильтруемом слое дисперсного материала затруднено в связи с тем, что он представляет собой гетерогенную двухкомпонентную систему «газ-твердые частицы», в которой на границе раздела компонентов скачкообразно изменяются физические свойства и параметры $[8,9$.$] . Поэтому при разработке методов расче-$ та слоевых аппаратов используют различные упрощенные модели, основанные на определенных представлениях. Достаточно обоснованными являются одно- и двухкомпонентные модели. В однокомпонентных моделях слой рассматриваются как квазисплошная среда с эффективными коэффициентами переноса. Такие модели, привлекающие своей простотой, могут быть использованы лишь в ограниченной области изменения параметров, когда температуры газового и твердого компонентов практически одинаковы. [10]. В ряде случаев (например, при наличии тепловыделения в слое, низкой интенсивности межкомпонентного теплообмена, кратковременных нестационарных процессах) температуры компонентов существенно различаются. В подобных условиях применяют двухкомпонентные модели, согласно которым слой рассматривается как система двух взаимопроникающих континуумов (твердого и газового компонентов) с соответствующими эффективными коэффициентами переноса. Процессы в каждом компоненте описываются уравнениями для сплошных сред, а взаимодействие между ними учитывается с помощью коэффициентов межкомпонентного теплообмена. Такие модели достаточно полно отражают основные особенности протекающих в слое процессов, в то же время сравнительно просты (несмотря на трудности в определении коэффициентов переноса для каждого компонента) [11].

\section{2. Математическая модель теплопереноса в плотном слое с внутренними источниками теп- лоты}

В работе рассматриваются взаимосвязанные процессы тепло- и массопереноса в слое гранулированного материала, через который продувается газ. Процессы переноса в такой системе могут быть описаны на основании двухкомпонентной гомогенной модели, согласно которой слой состоит из двух квазигомогенных компонентов - газового и твердого. Кондуктивный теплоперенос в компонентах характеризуется эффективными коэффициентами теплопроводности $\lambda_{2}^{*}, \lambda_{m}^{*}$ диффузионный перенос в газе - эффективным коэффициентом диффузии $D_{2}^{*}$, межкомпонентный тепло- и массообмен - коэффициентами тепло- и массоотдачи $\alpha_{M}, \beta_{M}$, теплообмен каждого из компонентов с погруженными поверхностями - коэффициентами теплоотдачи $\alpha_{c m}^{2}$, $\alpha_{c m}^{T}$.

Для упрощения модели внутренние термическое и диффузионное сопротивления переносу в частицах приняты пренебрежимо малыми по сравнению с внешними, что позволило исключить уравнения теплопроводности и диффузии в них. Однако при необходимости указанные сопротивления могут быть учтены путем внесения соответствующих поправок в значения коэффициентов межкомпонентного обмена $[10,12]$. Перенос теплоты в газовом компоненте, обусловленный массопереносом, также не учитывается.

При равномерном распределении по сечению порозности слоя, скорости газа, площади погруженных поверхностей поперечные градиенты температур и концентраций пренебрежимо малы по сравнению с продольными, вследствие чего задачу можно считать одномерной.

Математическая двухкомпонентная модель нестационарного тепломассопереноса в слое с погруженными поверхностями при протекании химической реакции во внешнедиффузионной области, полученная на основании законов сохранения и переноса энергии и массы, включает [13]:

- уравнение теплопереноса в газовом компоненте:

$$
\begin{gathered}
\rho_{2} c_{p_{z}} \varepsilon\left(1-\beta_{2}\right) \frac{\partial t_{2}}{\partial \tau}+\rho_{2} c_{p_{z}}\left(1-\beta_{1}\right) w_{2} \frac{\partial t_{2}}{\partial x}= \\
=\left(1-\beta_{1}\right) \frac{\partial}{\partial x}\left(\lambda_{z}^{*} \frac{\partial t_{2}}{\partial x}\right)+ \\
+\alpha_{M} a\left(1-\beta_{2}\right)\left(t_{m}-t_{2}\right)-\alpha_{c m}^{2} F_{c m}\left(t_{2}-t_{c m}\right)
\end{gathered}
$$

- уравнение теплопереноса в твердом компоненте:

$$
\begin{gathered}
\rho_{m} c_{m}(1-\varepsilon)\left(1-\beta_{2}\right) \frac{\partial t_{m}}{\partial \tau}=\left(1-\beta_{1}\right) \frac{\partial}{\partial x}\left(\lambda_{m}^{*} \frac{\partial t_{m}}{\partial x}\right)- \\
-\alpha_{M} a\left(1-\beta_{2}\right)\left(t_{m}-t_{2}\right)- \\
-\alpha_{c m}^{m} F_{c m}\left(t_{m}-t_{c m}\right)+r_{p} \beta_{M} a\left(1-\beta_{2}\right)\left(m_{2}-m_{m}\right),
\end{gathered}
$$

- уравнение массопереноса в газовом компоненте:

$$
\begin{aligned}
& \rho_{2} \varepsilon\left(1-\beta_{2}\right) \frac{\partial m_{2}}{\partial \tau}+\rho_{2}\left(1-\beta_{1}\right) w_{2} \frac{\partial m_{2}}{\partial x}= \\
& =\left(1-\beta_{1}\right) \frac{\partial}{\partial x}\left(D_{2}^{*} \rho_{2} \frac{\partial m_{2}}{\partial x}\right)- \\
& \quad-\beta_{M} a\left(1-\beta_{2}\right)\left(m_{2}-m_{m}\right)
\end{aligned}
$$

Начальные условия:

$$
\text { при } \tau=0 t_{m}=t_{m 0}, m_{2}=m_{20}
$$

Граничные условия:

$$
\begin{gathered}
\text { при } x=0 t_{m}=t_{m 0} t_{2}=t_{2}^{\prime}, m_{2}=m_{2}^{\prime} \\
\frac{\partial t_{m}}{\partial x}=\frac{\partial t_{2}}{\partial x}=\frac{\partial m_{2}}{\partial x}=0
\end{gathered}
$$


Здесь: $D_{2}^{*}$ - эффективный коэффициент диффузии газового компонента, м $^{2} / \mathrm{c} ; F_{c m}$ - удельная площадь погруженных поверхностей, м²/м $; m_{2}, m_{m}$ - безразмерные массовые концентрации реагента в газовом компоненте и на поверхности частиц; $\lambda_{2}{ }^{*}, \lambda_{m}{ }^{*}-$ эффективные аксиальные коэффициенты теплопроводности газового и твердого компонентов, Вт/(м·К).

Решение уравнений (1.1-1.3) с соответствующими условиями однозначности позволяет найти распределение по высоте слоя температур компонентов. Теплота фазовых переходов учитывается в уравнении теплопереноса в виде внутреннего источника теплоты.

Математическая модель таких процессов приобретает следующий вид.

- уравнение теплопереноса в газовом компоненте:

$$
\begin{gathered}
\rho_{2} c_{p_{2}} \varepsilon\left(1-\beta_{2}\right) \frac{\partial t_{2}}{\partial \tau}+\rho_{2} c_{p_{2}}\left(1-\beta_{1}\right) w_{2} \frac{\partial t_{2}}{\partial x}= \\
=\left(1-\beta_{1}\right) \frac{\partial}{\partial x}\left(\lambda_{2}^{*} \frac{\partial t_{2}}{\partial x}\right)+ \\
+\alpha_{M} a\left(1-\beta_{2}\right)\left(t_{m}-t_{2}\right)-\alpha_{c m}^{2} F_{c m}\left(t_{2}-t_{c m}\right),
\end{gathered}
$$

- уравнение теплопереноса в твердом компоненте:

$$
\begin{aligned}
& \rho_{m} c_{m}(1-\varepsilon)\left(1-\beta_{2}\right) \frac{\partial t_{m}}{\partial \tau}=\left(1-\beta_{1}\right) \frac{\partial}{\partial x}\left(\lambda_{m}^{*} \frac{\partial t_{m}}{\partial x}\right)- \\
- & \alpha_{M} a\left(1-\beta_{2}\right)\left(t_{m}-t_{2}\right)-\alpha_{c m}^{m} F_{c m}\left(t_{m}-t_{c m}\right)+ \\
+ & q_{v}\left(1-\beta_{2}\right)
\end{aligned}
$$

Начальное условие:

Граничные условия:

$$
\text { при } \tau=0 t_{m}=t_{m 0},
$$

$$
\text { при } x=0, t_{m}=t_{m 0} t_{2}=t_{2}^{\prime}, \frac{\partial t_{2}}{\partial x}=\frac{\partial t_{m}}{\partial x}=0
$$

В уравнение (1.7) входит производительность внутреннего источника теплоты $q_{v}$ определяется следующей зависимостью:

$$
q_{v}=\rho_{2} \frac{\partial m_{2}}{\partial \tau} r_{p}, \mathrm{BT} / \mathrm{M}^{3}
$$

Если принять допущение о пренебрежимо малой площади контакта твердого компонента с погруженными поверхностями, можно не учитывать теплообмен между ними, т.е. в уравнених (1.2), (1.7) считать $\alpha_{c m}^{m}=0$. При определенных условиях, вытекающих из сравнительной оценки порядка членов уравнений, можно пренебречь кондуктивным теплопереносом и диффузионным массопереносом:

$$
\begin{gathered}
\left(1-\beta_{1}\right) \frac{\partial}{\partial x}\left(\lambda_{2}^{*} \frac{\partial t_{2}}{\partial x}\right)=0, \\
\left(1-\beta_{1}\right) \frac{\partial}{\partial x}\left(\lambda_{m}^{*} \frac{\partial t_{m}}{\partial x}\right)=0 \\
\left(1-\beta_{1}\right) \frac{\partial}{\partial x}\left(D_{2}^{*} \rho_{2} \frac{\partial m_{2}}{\partial x}\right)=0
\end{gathered}
$$

При незначительном изменении эффективных коэффициентов теплопроводности и диффузии они могут быть приняты постоянными: $\lambda_{m}^{*}=$ const, $\lambda_{2}^{*}=$ const , $D_{2}^{*}=$ const. В ряде случаев и производительность внутренних источников теплоты может считаться неизменной ( $q_{v}=$ const $)$, либо зависящей от координаты: $\left(q_{v}=f(x)\right)$.

В определенной области изменения параметров, когда температуры компонентов практически одинаковы $\left(t_{2}=t_{m}=t\right)$, для продуваемого слоя применима однокомпонентная модель. Уравнение теплопереноса в слое имеет вид:

$$
\begin{gathered}
\rho_{c l} c_{c l}\left(1-\beta_{2}\right) \frac{\partial t}{\partial \tau}+\rho_{2} c_{2}\left(1-\beta_{1}\right) w_{2} \frac{\partial t}{\partial x}=\left(1-\beta_{1}\right) \frac{\partial}{\partial x}\left(\lambda^{*} \frac{\partial t}{\partial x}\right)+ \\
+\alpha_{c r} F_{c m}\left(t-t_{c m}\right)+q_{v}\left(1-\beta_{2}\right)
\end{gathered}
$$

Здесь $\rho_{c л}$ - плотность, $c_{c л}$ - теплоемкость, $\lambda^{*}$ - эффективный коэффициент аксиальной теплопроводности слоя; $\alpha_{c m}$ - коэффициент теплообмена слоя с погруженными поверхностями.

Начальное и граничное условия:

$$
\begin{gathered}
\text { при } \tau=0 t=t_{0}, \\
\text { при } x=0: t_{m}=t_{m 0} t=t^{\prime}
\end{gathered}
$$

Однокомпонентная модель применима при условияx:

$$
\gamma=\frac{\alpha_{c m}^{m} F_{c m}}{\alpha_{M} a\left(1-\beta_{2}\right)}<<1 ; \quad \varepsilon_{M}=\frac{\lambda_{m}^{*}\left(1-\beta_{1}\right)}{\alpha_{M} a\left(1-\beta_{2}\right) d_{m}^{2}}<<1,
$$

т.е. когда межкомпонентный теплообмен в слое значительно превышает теплоперенос за счет теплопроводности в твердом компоненте и теплообмен этого компонента с погруженными поверхностями.

Ниже приведены аналитические решения системы уравнений (1.6) - (1.7) и уравнения (1.11), которые позволяют выполнять приближенные расчеты с целью качественного и количественного анализа влияния режимных и геометрических факторов на распределение температур в слое [14-16]. Они также могут являться теоретической основой методик опытного определения коэффициентов теплообмена компонентов слоя с погруженными поверхностями.

При неизменных коэффициентах переноса система уравнений (1.6), (1.7) имеет вид [13]:

$$
\begin{gathered}
\rho_{2} c_{p_{2}} w_{2}\left(1-\beta_{1}\right) \frac{d v_{2}}{d x}=\lambda_{2}^{*}\left(1-\beta_{1}\right) \frac{d^{2} v_{2}}{d x^{2}}+ \\
+\alpha_{M} a\left(1-\beta_{2}\right)\left(v_{m}-v_{2}\right)-\alpha_{c m}^{2} F_{c m} v_{2}, \\
\lambda_{m}^{*}\left(1-\beta_{1}\right) \frac{d^{2} v_{m}}{d x^{2}}-\alpha_{M} a\left(1-\beta_{2}\right)\left(v_{m}-v_{2}\right)- \\
-\alpha_{c m}^{m} F_{c m} v_{m}-q_{v 0}(1 \pm b x)\left(1-\beta_{2}\right) \\
\text { при } x=0 v_{2}=v_{20}, \quad v_{m}=v_{m 0} \frac{d v_{2}}{d x}=\frac{d v_{m}}{d x}=0
\end{gathered}
$$

Здесь $q_{v 0}$ - производительность внутренних источников теплоты во входном сечении, Вт/м ${ }^{3}$.

При пренебрежимо малом кондуктивном теплопереносе в твердом компоненте (т.е. $\lambda_{m}^{*}\left(1-\beta_{1}\right) \frac{d^{2} v_{2}}{d x^{2}}=0$ ) температурное поле компонентов описывается следующими зависимостями [13].

$$
v_{2}=\frac{v_{20}}{P_{1}-P_{2}}\left[\left(P_{1}+\frac{1}{A_{1}}\right) \exp \left(P_{1} x\right)-\left(P_{2}+\frac{1}{A_{2}}\right) \exp \left(P_{2} x\right)\right]-
$$




$$
\begin{gathered}
-\frac{B_{1} H}{A_{1}\left(B_{2}+E_{2}\right)}\left[\frac{\exp \left(P_{1} x\right)}{P_{1}\left(P_{1}-P_{2}\right)}-\frac{\exp \left(P_{2} x\right)}{P_{2}\left(P_{1}-P_{2}\right)}+\frac{1}{P_{1} P_{2}}\right]+ \\
+\frac{B_{1} H b}{A_{1}\left(B_{2}+E_{2}\right)}\left[\frac{\exp \left(P_{1} x\right)}{P_{1}^{2}\left(P_{1}-P_{2}\right)}-\frac{\exp \left(P_{2} x\right)}{P_{2}^{2}\left(P_{1}-P_{2}\right)}+\frac{P_{1}+P_{2}}{P_{1}^{2} P_{2}^{2}}+\frac{x}{P_{1} P_{2}}\right] \\
v_{m}=\frac{\mathrm{v}_{2} B_{2}+H(1 \pm b x)}{B_{2}+E_{2}} \\
A_{1}=\frac{\lambda_{2}^{*}\left(1-\beta_{1}\right)}{G_{2} c_{p_{2}}} ; \\
B_{1}=\frac{\alpha_{M} a\left(1-\beta_{1}\right)}{G_{2} c_{p_{2}}} ; \\
E_{1}=\frac{\alpha_{c m}^{2} F_{c m}}{G_{2} c_{p_{2}}} ; E_{2}=\alpha_{M} a\left(1-\beta_{2}\right) \\
P_{1,2}^{m} F_{c m} ; H=-\frac{1}{2 A_{1}} \pm \sqrt{\frac{1}{4 A_{1}^{2}}-\frac{1}{A_{1}}\left(B_{1}+E_{1}\right)-\frac{B_{1} \cdot B_{2}}{B_{2}+E_{2}}}
\end{gathered}
$$

Из (1.32), (1.33) могут быть получены формулы для случаев, когда мощность источников неизменна (т.е. $b=0, q_{v}=q_{v 0}=$ const $)$, когда источники отсутствуют (т.е. $q_{v}=0, H=0$ ).

При значениях комплексов $\frac{E_{2}}{B_{2}}=\frac{\alpha_{c m}^{m} F_{c m}}{\alpha_{M} \cdot a\left(1-\beta_{2}\right)}<<1$ (когда термическое сопротивление межкомпонентного теплообмена значительно меньше, чем сопротивление теплообмена твердого компонента с погруженными поверхностями), и $\frac{H}{B_{2}}=\frac{q_{v 0}\left(1-\beta_{2}\right)}{\alpha_{M} a\left(1-\beta_{2}\right)}<<1$ (когда тепловой поток, обусловленный действием источников, значительно меньше, чем передаваемый в процессе межкомпонентного теплообмена), может быть использована однокомпонентная модель (1.11). В таких условиях при $\frac{\partial t}{\partial \tau}=0$ для распределения температур в слое справедлива формула:

$$
\begin{gathered}
\mathrm{v}=\frac{\mathrm{v}_{0}}{P_{1}-P_{2}}\left[\left(P_{1}+\frac{1}{A_{1}}\right) \exp \left(P_{1} x\right)-\left(P_{2}+\frac{1}{A_{2}}\right) \exp \left(P_{2} x\right)\right]- \\
\frac{H}{A_{1}}\left[\frac{\exp \left(P_{1} x\right)}{P_{1}\left(P_{1}-P_{2}\right)}-\frac{\exp \left(P_{2} x\right)}{P_{2}\left(P_{1}-P_{2}\right)}+\frac{1}{P_{1} P_{2}}\right]- \\
-\frac{H b}{A_{1}}\left[\frac{\exp \left(P_{1} x\right)}{P_{1}^{2}\left(P_{1}-P_{2}\right)}-\frac{\exp \left(P_{2} x\right)}{P_{2}^{2}\left(P_{1}-P_{2}\right)}+\frac{P_{1}+P_{2}}{P_{1}^{2} P_{2}^{2}}+\frac{x}{P_{1} P_{2}}\right] \\
A_{1}=\frac{\lambda^{*}\left(1-\beta_{1}\right)}{G_{2} c_{p_{2}}} ; E_{1}=\frac{\alpha_{c \Omega} F_{c m}}{G_{2} c_{p_{2}}} ; \quad H=\frac{q_{v 0}\left(1-\beta_{2}\right)}{G_{2} c_{p_{2}}} \\
P_{1}=-\frac{1}{2 A_{1}} \pm \sqrt{\frac{1}{4 A_{1}^{2}}-\frac{E_{1}}{A_{1}}}
\end{gathered}
$$

При неизменной мощности источников в (1.37) следует положить $b=0$, при их отсутствии $H=0$.

При значениях числа Пекле $P e^{*}=\frac{\rho_{2} c_{p_{2}} w_{2} D}{\lambda_{2}^{*}} \geq 50$ в двухкомпонентной модели можно пренебречь кондуктивным теплопереносом и в газовом компоненте, т.е. положить в (1.29) $\lambda_{2}^{*}\left(1-\beta_{1}\right) \frac{d^{2} v_{2}}{d x^{2}}=0 \cdot$ В этом случае распределение температур компонентов при неизменной мощности внутренних источников теплоты ( $q_{v}=$ const $)$ описывается формулами:

$$
\begin{gathered}
v_{2}=v_{20}\left[\exp (P x)+\frac{B_{1} H}{P\left(E_{2}+1\right) v_{20}}(1-\exp (P x))\right] \\
v_{m}=\frac{v_{2}+H}{1+E_{2}} \\
B_{1}=\frac{\alpha_{M} a\left(1-\beta_{2}\right)}{G_{2} c_{p_{2}}} \\
E_{1}=\frac{\alpha_{c m}^{2} F_{c m}}{G_{2} c_{p_{2}}} ; E_{2}=\frac{\alpha_{c m}^{m} F_{c m}}{\alpha_{M} a\left(1-\beta_{2}\right)} ; H=\frac{q_{v}}{\alpha_{M} a} \\
P=-\frac{B_{1}}{1+E_{2}}-B_{1}-E_{1}
\end{gathered}
$$

При отсутствии источников $\left(q_{v}=0\right)$ формулы (1.39), (1.40) переходят в (1.25), (1.26).

В однокомпонентной модели (1.11) кондуктивным теплопереносом в слое можно пренебречь при значениях числа Пекле $P e^{*} \geq 50$. В таких случаях температурное поле в слое при $q_{v}=$ const рассчитывается по формулам:

$$
\mathrm{v}=\mathrm{v}_{0}\left[\exp (P x)-\frac{H}{P \mathrm{v}_{20}}(1-\exp (P x))\right]
$$

где

$$
P=-\frac{\alpha_{c л} F_{c m}}{G_{2} c_{p_{2}}}, \quad H=\frac{q_{v}\left(1-\beta_{2}\right)}{G_{2} c_{p_{2}}}
$$

При $q_{v}=0$ (1.44) переходит в (1.28).

\section{3. Аналитическое исследование температур- ных полей газового и твердого компонентов}

По приведенным выше аналитическим зависимостям были выполнены расчеты стационарных температурных полей при различных режимных и геометрических характеристиках. Необходимые в расчетах коэффициенты теплообмена компонентов слоя с погруженными поверхностями определялись по рекомендациям [16], межкомпонентного теплообмена - по [12]. Выполнены расчеты по двум модификациям двухкомпонентной модели, различающимся способом учета теплообмена с погруженными поверхностями:

- в модификации 1 учитывается теплообмен каждого из компонентов с помощью соответствующих коэффициентов теплоотдачи $\alpha_{c m}^{2}, \alpha_{c m}^{m}$;

- в модификации 2 теплообмен твердого компонента считается пренебрежимо малым, учитывается только теплоотдача газового компонента с помощью величины $\alpha_{c m}^{2 *}=\alpha_{c m}^{2}+\alpha_{c m}^{m}$.

Проводилось количественное сопоставление результатов расчетов по обеим модификациям, для оценки расхождения использовали величину относительной 
разности расчетных температур газового и твердого компонентов $\delta_{m}=\frac{t_{m 1}-t_{m 2}}{t_{m 1}} ; \delta_{2}=\frac{t_{21}-t_{22}}{t_{21}}$.

Расчеты велись для различных скоростей газа $(0,05$ - 0,5) м/с, размеров частиц $(0,002-0,01)$ м, их теплопроводности $(0,75-11) \mathrm{BT} /($ м $\cdot$ К), чисел Рейнольдса $(10$ - 1000) при $q_{v}=0$.

На рис. 1 представлены распределения температур по высоте слоя по модификации 1 для двух режимов. Модификация 2 дает качественно такие же результаты при определенных количественных расхождениях. Анализ показывает, что на величины $\delta_{m}, \delta_{2}$ существенно влияет число Рейнольдса. Так, при $d=9,3$ мм, $\lambda_{m}=11 \mathrm{BT} /(\mathrm{м} \cdot \mathrm{K}), x=0,5$ м, при $R e=50-\delta_{2}=5,7 \%$, $\delta_{m}=14,3 \%$, а при $R e=350-\delta_{2}=1 \% ; \delta_{m}=5,8 \%$. По мере увеличения диаметра частиц расхождение между моделями растет. Так, при $R e=100, x=0,17$ м, изменение диаметра с 2,95 мм до 9,3 мм приводит к росту $\delta_{2}$ от $1,4 \%$ до $3,3 \%, \delta_{m}$ - от $3,2 \%$ до $10,5 \%$. С ростом теплопроводности материала частиц расхождение также увеличивается. Так, при $R e=100, x=0,17$ м, $d=2,95$ м изменение $\lambda_{m}$ с 2 до $11 \mathrm{BT} /($ м $\cdot К)$ ведет к увеличению $\delta_{2}$ от 0,5 до $1,4 \%, \delta_{m}$ - от 2,2 до $3,3 \%$.

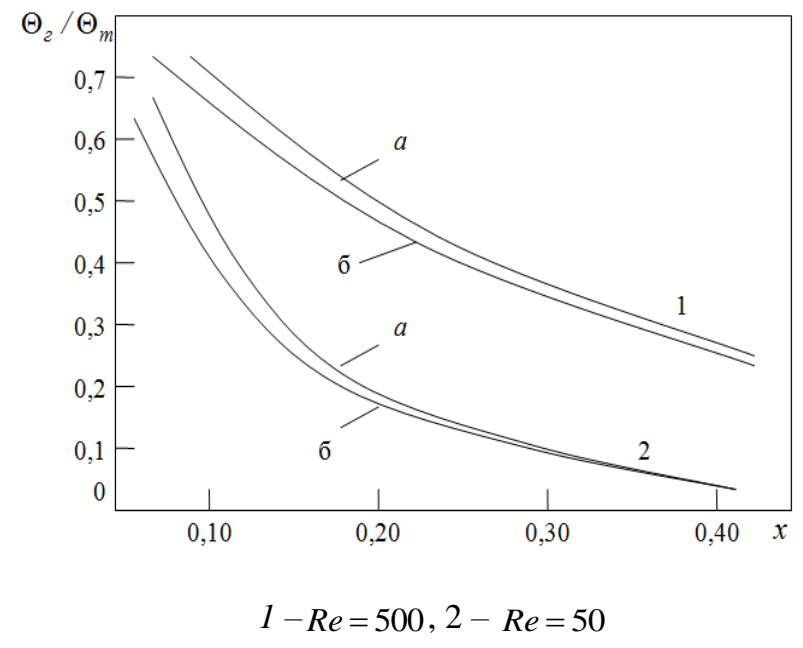

Рисунок 1 - Поля температур газового (a) и твердого (б) компонентов слоя при $d_{m}=9,3 \mathrm{MM}$,

$$
\lambda_{m}=11 \mathrm{BT} /(\mathrm{M} \cdot \mathrm{K}) \text {. }
$$

Таким образом, при фиксированной геометрии погруженных поверхностей расхождение температур, рассчитанных по обеим модификациям модели, зависит от числа Рейнольдса, свойств компонентов, продольной координаты.

Критерием сходимости может служить комплекс $\gamma=\frac{\alpha_{c m}^{m} F_{c m}}{\alpha_{M} a\left(1-\beta_{2}\right)}($ либо $1 / \gamma)$, учитывающий эти факторы.

На рис. 2 представлены зависимости относительных разностей температур от $1 / \gamma$. Величины $\delta_{2}, \delta_{m}$ в области малых значений комплекса $1 / \gamma$ заметно снижаются с его увеличением, при $1 / \gamma>12$ темп зависимости уменьшается, а при $1 / \gamma>30$ наблюдается практическая автомодельность. В рассмотренном диапазоне изменения $1 / \gamma$ значения $\delta_{m}$ составляют $(4-20) \%, \delta_{2}(1-$ $10) \%$, при $1 / \gamma=$ idem,$\delta_{m}>\delta_{2}$.

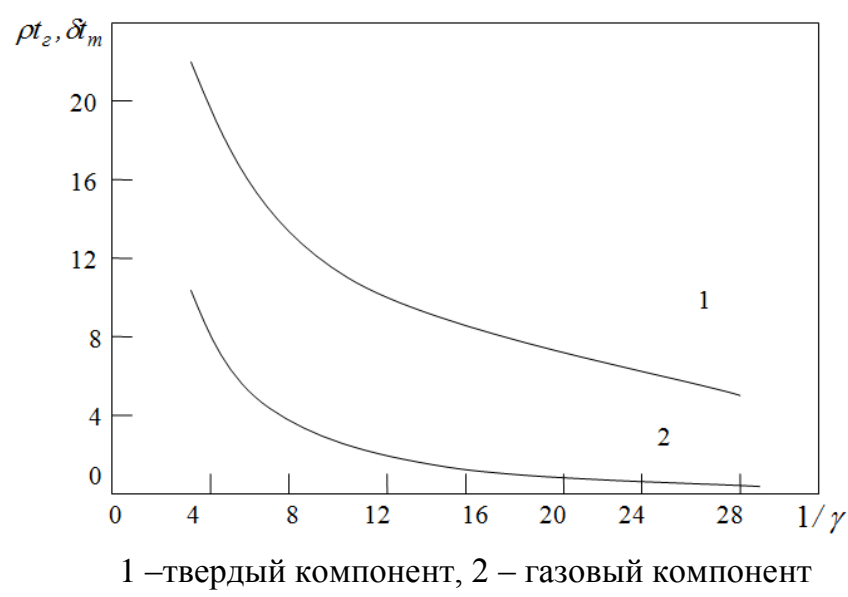

Рисунок 2 - Влияние комплекса $1 / \gamma$ на расхождение температур при $\lambda=11 \mathrm{~B} /($ м $\cdot \mathrm{K}), R e=50, d_{m}=9,3$ мм,

$$
x=0,17 \text { м. }
$$

Условие $1 / \gamma>30$ (или $\gamma<0,03$ ) выполняется при высоких числах Рейнольдса, малых $\lambda_{m}, d_{m}, x$, когда термическое сопротивление межкомпонентного теплообмена мало по сравнению с сопротивлением теплоотдачи от твердого компонента к стенке. Выполнение этого условия обеспечивает удовлетворительное согласование температур, рассчитанных по обеим модификациям моделей.

На рис. 3 сопоставлены экспериментальные распределения температур компонентов в слое с расчетными (для условий опытов обе модификации модели дают практически одинаковые результаты). Сплошные и пунктирные кривые - расчетные температуры газа и частиц соответственно, точки - экспериментальные температуры газа и частиц.

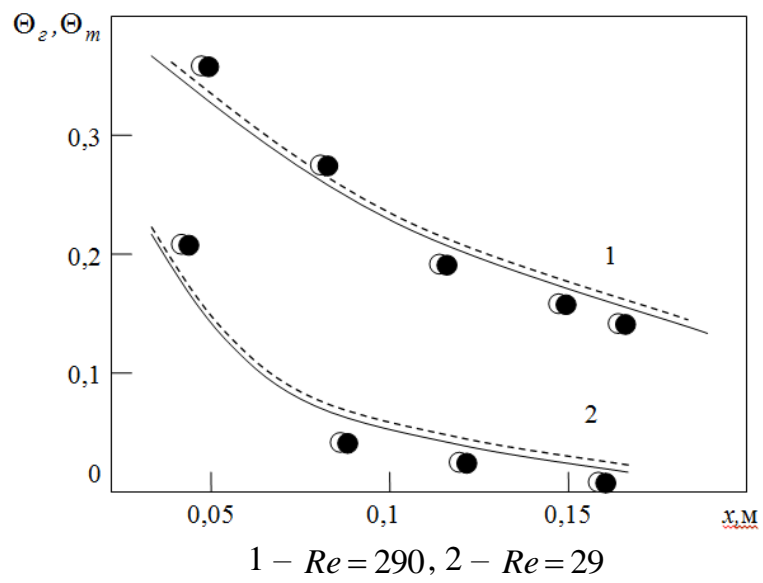

Рисунок 3 - Сопоставление экспериментальных и расчетных полей температур в слое при $D=0,0335$ м, $S_{1} / D=2, S_{2} / D=2, d=3 \mathrm{мм}, \lambda_{m}=0,756 \mathrm{BT} /(\mathrm{м} \cdot \mathrm{K})$. 
Удовлетворительное качественное и количественное согласование экспериментальных и расчетных данных свидетельствует о том, что модели верно описывают основные закономерности теплопереноса в продуваемом слое с погруженными теплообменными поверхностями.

Был проведен анализ чувствительности моделей к погрешностям определения коэффициентов теплоотдачи компонентов $\alpha_{c m}^{m}, \alpha_{c m}^{2}, \alpha_{c m}^{2 *}$. Установлено, что эта погрешность существенно влияет на значения расчетных температур. Так, например, при $R e=25$, $\lambda_{m}=0,75 \mathrm{BT} /(\mathrm{м} \cdot \mathrm{K}), d_{m}=2,95$ мм, $x=0,17$ м, при изменении $\alpha_{c m}^{2}$ и $\alpha_{c m}^{m}$ на $10 \%$ и на $20 \%$ температуры компонентов меняются на $14 \%$ и $31 \%$ соответственно. Таким образом, неточность в определении коэффициентов теплоотдачи приводит к существенным ошибкам в расчете температурных полей. Эти данные позволяют сформулировать требования к точности опытных данных. Эксперименты значительно упрощаются и дают меньшую погрешность при определении коэффициента теплоотдачи $\alpha_{c m}^{2 *}$, чем $\alpha_{c m}^{2}$ и $\alpha_{c m}^{m}$. Поэтому при соблюдении условия $\gamma<<0,03$ удобнее применять вторую модификацию расчетной модели, определив из экспериментов $\alpha_{c m}^{2 *}$. Если же это условие не выполняется, следует применять первую модификацию модели с использованием опытных значений $\alpha_{c m}^{2}, \alpha_{c m}^{m}$ для каждого из компонентов. Итак, при расчетах температурных полей необходимы надежные сведения о коэффициентах слоя и его компонентов.

\section{Выводы}

Полученные на основании аналитического решения двухкомпонентной математической модели в одномерном приближении зависимости позволяют качественно верно оценить характер распределения температур компонентов по высоте слоя в широком диапазоне изменения входных величин. Представленные модели верно описывают основные закономерности теплопереноса в продуваемом слое с погруженными теплообменными поверхностями Анализ чувствительности моделей показал, что погрешность определения коэффициентов теплоотдачи компонентов $\alpha_{c m}^{m}, \alpha_{c m}^{2}, \alpha_{c m}^{2 *}$ существенно влияет на значения расчетных температур: при $R e=25, \quad \lambda_{m}=0,75 \mathrm{BT} /(\mathrm{M} \cdot \mathrm{K}), d_{m}=2,95 \quad \mathrm{MM}$, $x=0,17$ м, при изменении $\alpha_{c m}^{2}$ и $\alpha_{c m}^{m}$ на $10 \%$ и на $20 \%$ температуры компонентов меняются на $14 \%$ и $31 \%$ соответственно.

\section{Литература}

1. Денисов-Винский Н. Д. Вторичные энергетические ресурсы как резерв энергосбережения. Энергобезопасность. 2008. №2 (20). С. 23-28.
2. Jacques D. Sands. Powders, and Grains: An Introduction to the Physics of Granular Materials. New York: Springer, 2012. $214 \mathrm{p}$

3. Optimization and economic evaluation of industrial gas production and combined heat and power generation from gasification of corn stover and distillers grains. Kumar, A. H. et al. Bioresource Technol. 2010. №101. C. 3696-3701.

4. Goldhirsch I. Rapid granular flows. Fluid Mechanic. 2003. Vol. 35. P. 267-293

5. Study on performance of the ball packed-bed regenerator: experiments and simulation / J.Yu et al. Applied Thermal Engineering. 2014. Vol. 69. P.113-122.

6. Li J., Mason D. J. Application of the discrete element modeling in air drying of particulate solids. Technology. 2002. Vol. 20, №3. Р. 255-282.

7. Саваторова В. Л., Белый А. А. Математическое моделирование процессов кондуктивной теплопередачи в гетерогенных средах с периодической структурой. Москва, 2010. $100 \mathrm{c.}$

8. Sadrameli S. M., Ajdari H. R. B. Mathematical modeling and simulation of thermal regenerators including solid radial conduction effects. Applied Thermal Engineering. 2015. Vol. 76. 441-446.

9. Adeyanju A. A., Manohar K. Theoretical and Experimental Investigation of Heat Transfer in Packed Beds. Research Journal of Applied Sciences. 2009. Vol. 4, №5. P. 166-177.

10. Горбис 3. Р., Календерьян В. А. Теплообменники с проточными дисперсными теплоносителями. Москва, $1975.296 \mathrm{c.}$

11. Процессы и аппараты химической технологии: Ч. 1. Теоретические основы процессов химической технологии. Гидромеханические и тепловые процессы и аппаpambl. Халил В. Б., и др. Новополоцк: УО «ПГУ», 2006. $328 \mathrm{c}$.

12. Аэров М. Э., Тодес О. М., Наинский Д. А. Аппараты с стационарным зернистым слоем. Гидравлические и тепловые основы работы. Львов, 1979. 176 с.

13. Календерьян В. А., Бошкова И. Л. Тепломассоперенос в аппаратах с плотным дисперсным слоем. Киев: Слово, 2011. 184 с.

14. Календерьян В.А., Гаппасов В.Р., Ахмед Султан. Температурное поле в неподвижном фильтруемом слое с источниками тепла и погруженными поверхностями. Математические методы теплопереноса. Днепропетровск, 1986. с.55-58.

15. Календерьян В. А., Гаппасов В. Р., Овчаренко О. Л. Тепломассоперенос в неподвижном фильтруемом слое с погруженными поверхностями. Повышение эффективности, совершенствование процессов в аппаратах химич. Производств: материалы VIII респ. конф. Днепропетровск, 1991. С.60-61

16. Календерьян В. А., Гаппасов В. Р., Овчаренко О. Л. Об учете теплообмена неподвижного фильтруемого слоя с погруженными поверхностями в двухкомпонентной модели теплопереноса. Инженерно-физический журнал, 1992, Т.63, №1, С. 63-68.

Отримана в редакції 06.02.2018, прийнята до друку 06.03.2018 


\title{
Analytical study of heat transfer in dense layer of granulated material with internal sources of heat
}

\author{
I. L. Boshkova, A. V. Solodkaya, O. S. Bodiul, E. I. Altman \\ Odessa National Academy of Food Technologies, 112 Kanatnaya St., Odessa, 65039, Ukraine
}

\begin{abstract}
The results of an analytical study of heat and mass transfer in a dense layer of granular material with a gas stream as a heat exchange region of regenerative and recuperative devices are presented. It is noted that the determination of rational operating parameters of thermal engineering devices depends on an adequate mathematical model of heat exchange between gas flows and granular material and reliable data for the coefficients of intercomponent heat exchange, for which additional experimental e study. A rigorous description of the transport processes in a dense, stationary, filtered layer of dispersed material is difficult due to the fact that it is a heterogeneous two-component gassolid particle system in which the physical properties and parameters change discontinuously at the interface of the components. A mathematical model of heat exchange between a dense layer of particles and a gas stream is presented, which is obtained on the basis of a two-component homogeneous model with allowance for the effect of internal heat sources in the one-dimensional approximation. An analytical study of the temperature fields of the gas and solid components under various regime and geometric characteristics has shown a satisfactory agreement between the calculated and experimental data, but the sensitivity of the model to the given values of the heat transfer coefficients should be taken into account, the inaccuracy of which may lead to significant errors in the calculation of the temperature fields.
\end{abstract}

Keywords: Mathematical Model; Heat Exchange; Granular Layer; One-Dimensional Approximation; Temperature Dependences; Internal Sources; Calculation of Temperature Fields.

\section{References}

1. Denisov-Vinskij N. D. 2008. Vtorichnye ehnergeticheskie resursy kak rezerv ehnergosberezheniya. Energobezopasnost, no.2 (20), 23-28. (in Russian).

2. Jacques D. 2012. Sands, Powders, and Grains: An Introduction to the Physics of Granular Materials. New York: Springer, $214 \mathrm{p}$.

3. Kumar, A. H. et al. 2010. Optimization and economic evaluation of industrial gas production and combined heat and power generation from gasification of corn stover and distillers grains. Bioresource Technol, no.101, 3696-3701. DOI: https://doi.org/10.1016/j.biortech.2009.12.103

4. Goldhirsch I. 2003. Rapid granular flows. Fluid Mechanic, vol. 35, 267-293. DOI: https://doi.org/10.1146/ annurev.fluid.35.101101.161114

5. J.Yu et al. 2014. Study on performance of the ball packed-bed regenerator: experiments and simulation. Applied Thermal Engineering, vol. 69, 113-122.

6. Li J., Mason D. J. 2002. Application of the discrete element modeling in air drying of particulate solids. Drying Technology, vol. 20, iss. 3, 255-282. DOI: https://doi.org/10.1081/drt-120002542

7. Savatorova V. L., Belyj A. A. 2010. Matematicheskoe modelirovanie processov konduktivnoj teploperedachi $\mathrm{v}$ geterogennyh sredah s periodicheskoj strukturoj. Moskva, 100 p. (in Russian).

8. Sadrameli S. M., Ajdari H. R. B. 2015. Mathematical modeling and simulation of thermal regenerators including solid radial conduction effects. Applied Thermal Engineering, vol. 76, 441-446.

DOI: https://doi.org/10.1016/j.applthermaleng.2014.11.035 9. Adeyanju A. A., Manohar K. 2009. Theoretical and Experimental Investigation of Heat Transfer in Packed Beds. Research Journal of Applied Sciences, vol. 4, iss. 5, 166-177.
10. Gorbis Z. R., Kalender'yan V. A. 1975. Teploobmenniki s protochnymi dispersnymi teplonositelyami. Moskva, 296 p. (in Russian).

11. Processy i apparaty himicheskoj tekhnologii: CH. 1. Teoreticheskie osnovy processov himicheskoj tekhnologii. Gidromekhanicheskie i teplovye processy i apparaty. Halil V. B., i dr. Novopolock: UO «PGU», 2006. 328 p. (in Russian).

12. Aerov M. E., Todes O. M., Nainskij D. A. 1979. Apparaty s stacionarnym zernistym sloem. Gidravlicheskie i teplovye osnovy raboty. L'vov, 176 p. (in Russian).

13. Kalender'yan V. A., Boshkova I. L. 2011. Teplomassoperenos $\mathrm{v}$ apparatah s plotnym dispersnym sloem. Kiev: Slovo, 184 p. (in Russian).

14. Kalender'yan V.A., Gappasov V.R., Ahmed Sultan. 1986. Temperaturnoe pole $\mathrm{v}$ nepodvizhnom fil'truemom sloe s istochnikami tepla i pogruzhennymi poverhnostyami. Matematicheskie metody teploperenosa. Dnepropetrovsk, P. 55-58 (in Russian).

15. Kalender'yan V.A., Gappasov V.R., Ovcharenko O.L. 1991. Teplomassoperenos V nepodvizhnom fil'truemom sloe s pogruzhennymi poverhnostyami. Povyshenie ehffektivnosti, sovershenstvovanie processov v apparatah himich. Proizvodstv: materialy VIII resp. konf. Dnepropetrovsk, P. 60-61 (in Russian).

16. Kalender'yan V.A., Gappasov V.R., Ovcharenko O.L. 1992. Ob uchete teploobmena nepodvizhnogo fil'truemogo sloya $\mathrm{s}$ pogruzhennymi poverhnostyami $\mathrm{v}$ dvuhkomponentnoj modeli teploperenosa. Inzhenernofizicheskij zhurnal, T.63, no.1, P.63-68 (in Russian). 